\title{
Synergistic Inhibition of Breast Cancer Cell Growth by an Epigenome- Targeting Drug and a Tyrosine Kinase Inhibitor
}

\author{
Yu Shan Wu, ${ }^{a}$ Yuan Quan, ${ }^{b}$ Dong Xing Zhang, ${ }^{a}$ Dong Wu Liu, ${ }^{a}$ and Xiu Zhen Zhang, ${ }^{*}$, \\ ${ }^{a}$ School of Life Sciences, Shandong University of Technology; Zibo 255049, P. R. China: and ${ }^{b}$ Agricultural \\ Bioinformatics Key Laboratory of Hubei Province, College of Informatics, Huazhong Agricultural University; \\ Wuhan 430070, P. R. China. \\ Received May 1, 2017; accepted July 10, 2017
}

\begin{abstract}
Background: Epigenome-targeting drugs, for example, histone decetylases (HDACs) inhibitors, have been recently shown to induce apoptosis in a variety of cancer cells, which could potentially be used as anticancer therapy. Tyrosine kinase inhibitors (TKIs) have been widely used in clinical trials of various cancers. HDAC inhibitor vorinostat, TKIs dasatinib have been tested in pivotal phase 2 clinical trials in patients with breast cancer. The combination treatment of vorinostat with dasatinib is expected to have synergistic effect on inhibiting breast cancer cell growth. Materials and Methods: Antiproliferation effects of the combined drugs on MCF-7 cells were designed according to Chou-Talalay method and analyzed with 3-(4,5-dimethylthiazol-2-yl)-2,5-diphenyltetrazolium bromide (MTT) assay. Cell-cycle perturbation and cell apoptosis induction of the combination drugs were examined by Flow cytometry. The generation of reactive oxygen species (ROS), loss of mitochondrial membrane potential, and the expression of Bcl-2 were determined by Western blot. Results: Our results revealed that the combination treatment had synergistic effects on anti-MCF7 cells, enhanced $\mathrm{G}_{2} / \mathrm{M}$ cell arrest, the generation of ROS, the loss of mitochondrial membrane potential, and cell apoptosis in MCF-7 cells in synergy. Moreover, the combination treatment decreased Bcl-2 expression. Conclusion: Our results demonstrated that the combination of vorinostat with dasatinib exerted synergistic anticancer effects on MCF-7 cells by inducing cell cycle arrest, ROS production, and apoptosis through the mitochondria-mediated intrinsic pathway.
\end{abstract}

Key words vorinostat; dasatinib; breast cancer; apoptosis; combination therapy

Breast cancer is one of the most common malignancies in women and has become a major cause of women's death worldwide. ${ }^{1)}$ Breast cancer occurs in the mammary epithelium or ductal epithelium. In recent years, the pathogenesis of breast cancer has been continuously investigated by medical researchers. ${ }^{2}$ As a result, many drugs for treatment of breast cancer have been marketed, including natural ingredients (such as paclitaxel and vincristine), chemical drugs (such as capecitabine and tamoxifen) and targeted drugs (such as Herceptin and lapatinib). Although these drugs have been widely used in the treatment of breast cancer, the serious side effects (especially for chemical drugs) and drug resistance in patients (especially for targeted drugs) also limited their clinical use. ${ }^{3-5)}$

It is well known that most human complex diseases, especially cancer, are not the result of a single disease gene, but are caused by complex biological networks. ${ }^{6}$ As a result, it is reasonable to speculate that targeting multiple disorder factors of disease will have higher medicinal potential than single-targeted agents. In the context of cancer, drug combinations may target multiple pathways and significantly inhibit the growth of tumor cells, and will be more conducive to treating cancer than single therapeutic agents. ${ }^{7}$ As for breast cancer, drug combinations (such as vinorelbine-cisplatin and capecitabinevinorelbine) are indeed commonly used in clinical treatment and have shown excellent results. ${ }^{8,9)}$

With the development of next-generation sequencing technology in the past decade, epigenetic processes have been found to play a key role in the causation, progression and treatment of cancer. ${ }^{10)}$ The synthesis of small molecules that can target the mutated or dysregulated epigenetic modifiers and alter epigenomes in cancers represents one of novel strate- gies for cancer therapy. ${ }^{11)}$ For example, histone deacetylase (HDAC) can "erase" acetylation marks on histones, which is crucial in epigenetic processes of cancer. ${ }^{12,13)}$ Previous studies have found that in a variety of cancers, HDACs have abnormal activity and expression. ${ }^{12)}$ And epigenome-targeting drug vorinostat (also known as suberoylanilide hydroxamic acid) is a direct HDAC inhibitor that has been approved for clinical trials of various cancers, such as $\mathrm{T}$ cell lymphomas. ${ }^{11)}$ In addition, vorinostat has been tested in a pivotal phase 2 clinical trial in patients with breast cancer (https://clinicaltrials.gov/).

Tyrosine kinase is also a hot spot of cancer therapeutic target. For instance, the second-generation c-abl oncogene (ABL) tyrosine kinase inhibitors (TKIs) (e.g., imatinib, ponatinib and dasatinib) are Food and Drug Administration (FDA)approved drugs for chronic myelogenous leukemia treatment (DrugBank). Combination of ABL TKIs and vorinostat has shown potential for treating leukemia. ${ }^{14)}$ Considering the fact that the dasatinib have phase 2 clinical records of anti-breast cancer in clinical trials, we speculated that combining dasatinib with vorinostat may have synergistic effects for breast cancer. This stimulated our interest to test this hypothesis by experiments.

\section{MATERIALS AND METHODS}

Cells and Reagents The human breast cancer cell line MCF-7 was obtained from China Center for Type Culture Collection (Wuhan, Hubei, China). The cells were maintained in an RPMI-1640 medium (Gibco, U.S.A.) supplemented with $10 \%$ newborn bovine serum in a humidified $5 \% \mathrm{CO}_{2}$ incubator at $37^{\circ} \mathrm{C}$. Vorinostat and dasatinib (purity $>98 \%$ ) were 

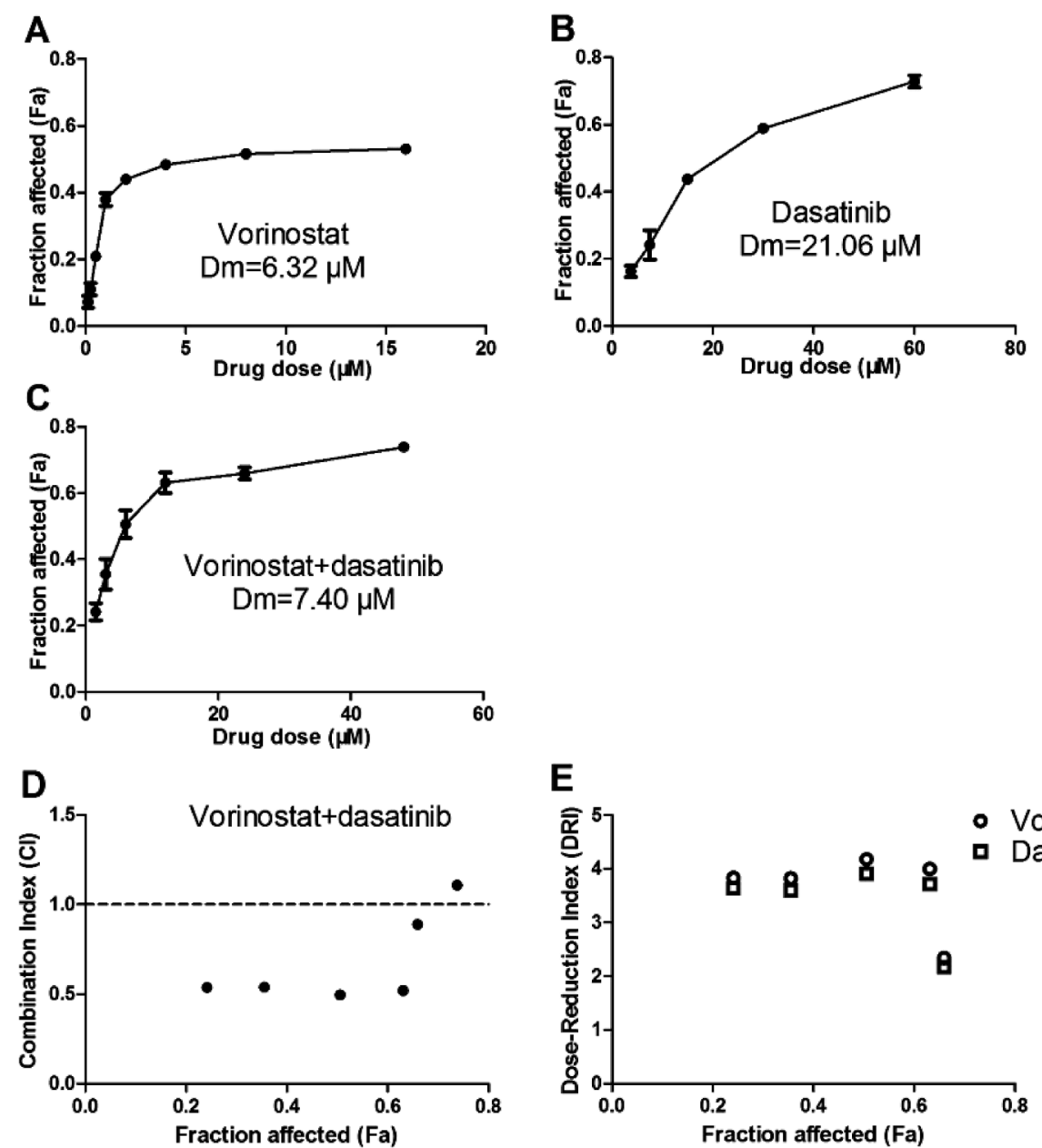

Fig. 1. Synergistic Anticancer Effects of the Combination of Vorinostat with Dasatinib in MCF-7 Cells

Cells were treated with vorinostat (A) and dasatinib (B), alone and in combination (C) for $48 \mathrm{~h}$. The viabilities of cells were qualified by MTT assay, and reported as fraction affected (fraction of cells affected by treatment), and the data represented the mean \pm S.D. from five replicate experiments. The combination indexes (CIs) (D) and dose-reduction indexes (DRIs) (E) of the combinations of vorinostat with dasatinib were calculated as described. The data points below CI values of 1 , denoted by horizontal dotted lines in (D), are indicative of synergistic effects.

purchased from Haoyuan Chemexpress Co., Ltd. (Shanghai, China).

Cell Viability Assay Cell viability was determined via 3-(4,5-dimethylthiazol-2-yl)-2,5-diphenyltetrazolium bromide (MTT) assay (Sigma-Aldrich, U.S.A.). ${ }^{15)}$ The cells were seeded onto 96 -well plates $\left(6 \times 10^{3}\right.$ cell/well), and then incubated overnight. The cells were then treated with vorinostat and dasatinib alone or in combination for $48 \mathrm{~h}$, with $0.1 \%$ dimethyl sulfoxide (DMSO) as negative control. Approximately $10 \mu \mathrm{L}$ of the $5 \mathrm{mg} / \mathrm{mL}$ MTT solution was added to each well, and the plates were incubated for more $4 \mathrm{~h}$ at $37^{\circ} \mathrm{C}$. Finally, the absorbance was detected at $492 \mathrm{~nm}$ using a Thermo Multiskan MK3 (Thermo Fisher, U.S.A.). $\mathrm{IC}_{50}$ was determined based on absorbance readings. The ratios of drug combination were based on the ratios of $\mathrm{IC}_{50}$. All samples were tested in five replicates, and the experiments were repeated at least three times.

The interaction between two drugs was analyzed according the median-effect principle proposed by Chou and Talalay. ${ }^{16,17)}$ The median effect dose of the drug combinations equation, Dm, (analogous to the $\mathrm{IC}_{50}$ ) and combination index (CI) were calculated by CompuSyn software and CI value below 1 represents synergism. Dose-reduction index (DRI) was used to measure the number of reduction folds in the dose may be produced by the synergistic combination of the two drugs at a given effect level compared with the doses of each drug alone. ${ }^{17)}$

Cell Cycle and Cell Apoptosis by Flow Cytometry Cell cycle and apoptosis were detected by flow cytometry. The cells were seeded onto 6-well plates and cultured with vorinostat $(1.5 \mu \mathrm{M})$ and dasatinib $(4.5 \mu \mathrm{M})$ alone and in combination for $48 \mathrm{~h}$. Then, the detached/dead and viable cells were collected. Apoptosis was determined by staining with Annexin V-fluorescein isothiocyanate (FITC) according the kit instruction. Cells for cell cycle analysis were washed with cold phosphate buffered saline (PBS), fixed with 70\% ethanol, incubated with RNAase and stained with propidium iodide (PI). Finally, the cells were analyzed by a FACSCalibur flow cytometer equipped with the CellQuest and Modifit software (Becton Dickinson, U.S.A.).

Reactive Oxygen Species (ROS) Detection Intracellular ROS generation was evaluated by using the oxidationsensitive fluorescent probe $2^{\prime}, 7^{\prime}$-dichloro-fluorescin diacetate according to the method of Engelmann et al.. ${ }^{18)}$ Briefly, the cells $\left(1 \times 10^{5} / \mathrm{mL}\right)$ were seeded on cover slips and cultured with vorinostat $(1.5 \mu \mathrm{M})$ and dasatinib $(4.5 \mu \mathrm{M})$, alone and in combination, for $12 \mathrm{~h}$. The cells were then washed with PBS and incubated with $2^{\prime}, 7^{\prime}$-dichlorofluorescin diacetate (DCFHDA) $(100 \mu \mathrm{L}, 10 \mu \mathrm{M})$ for $30 \mathrm{~min}$ in the dark. Fluorescence was detected by a confocal microscope (Leica TCS SP2) with 

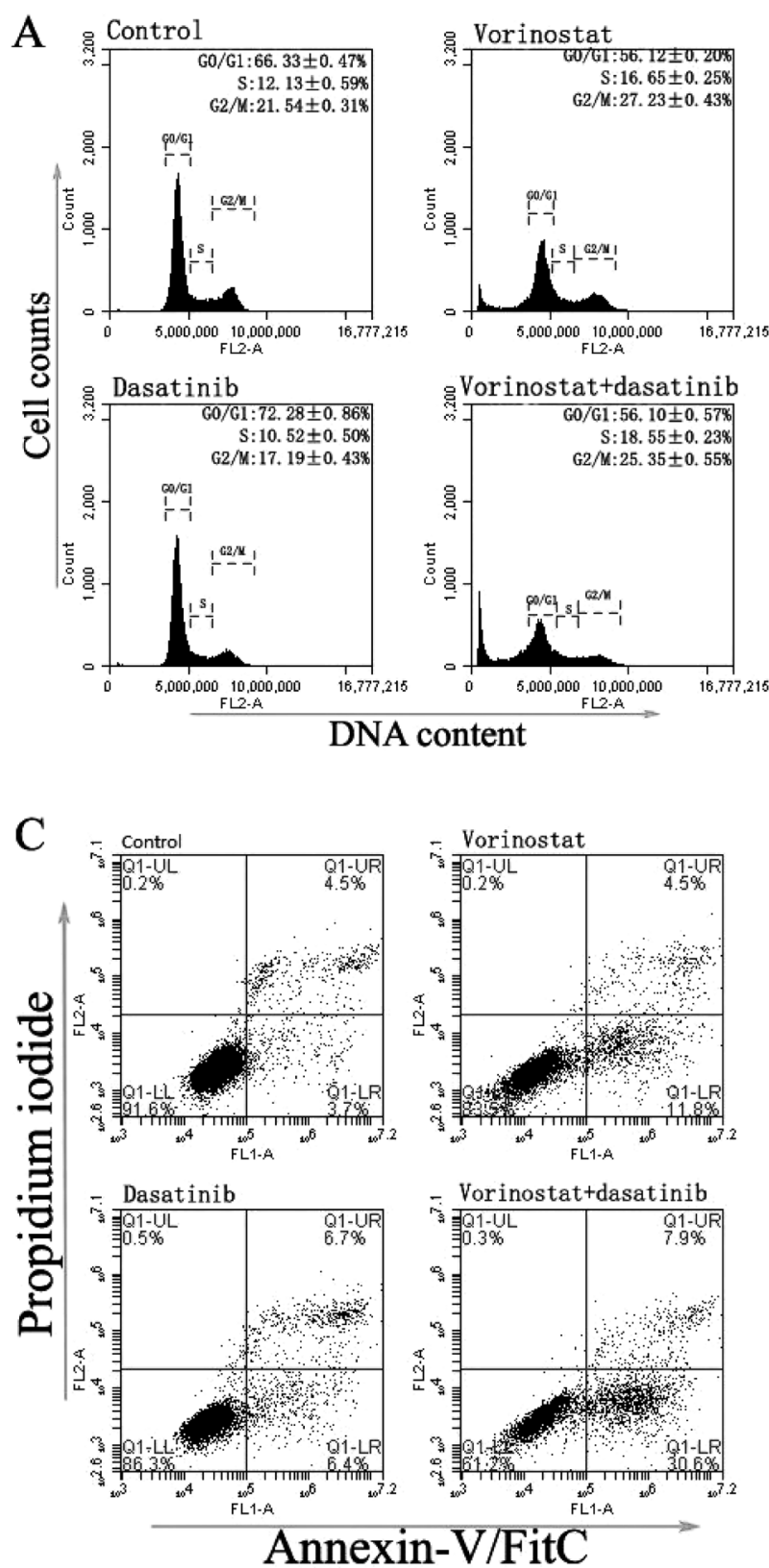

B

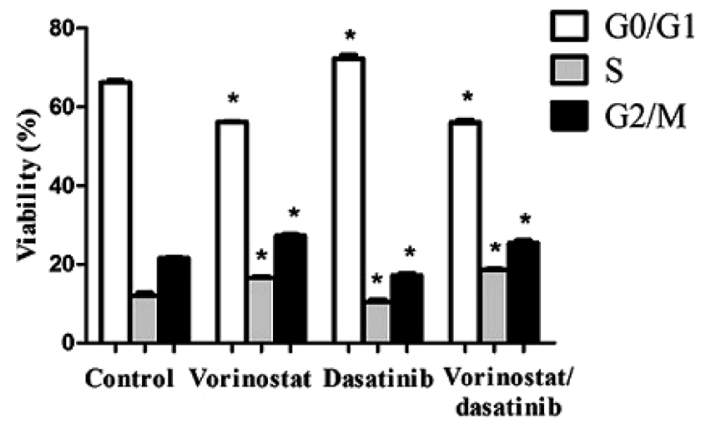

D

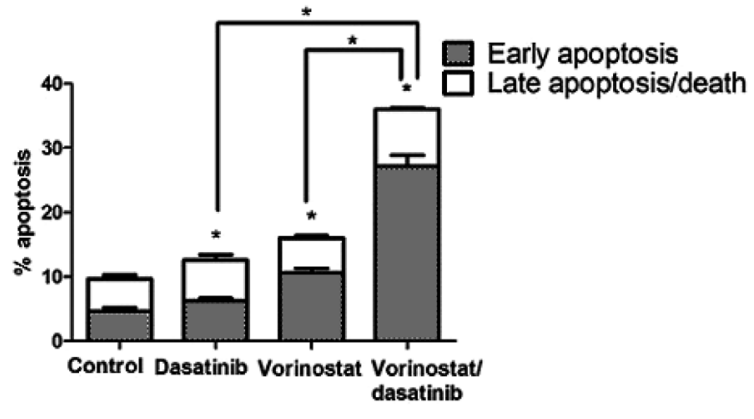

Fig. 2. Synergistic Induction of $\mathrm{G}_{2} / \mathrm{M}$ Arrest and Cell Apoptosis by the Combination of Vorinostat with Dasatinib in MCF-7 Cells

(A and B) Cells were treated with vorinostat $(1.5 \mu \mathrm{M})$ and dasatinib $(4.5 \mu \mathrm{M})$, alone and in combination for $12 \mathrm{~h}$ and cell cycle distribution was examined using PI staining followed by flow cytometric analysis. (C and D) Cells were treated with vorinostat $(1.5 \mu \mathrm{M})$ and dasatinib $(4.5 \mu \mathrm{M})$, alone and in combination for $12 \mathrm{~h}$ and cell apoptosis was examined using Annexin V/PI staining followed by flow cytometric analysis. The numerical data were presented as mean \pm S.D. $(n=3)$. ${ }^{*}$ Indicates a significant difference $(p<0.05)$ by one-way ANOVA.

$100 \times$ objective at an excitation wavelength $488 \mathrm{~nm}$ and an emission wavelength of $530 \mathrm{~nm}$. The fluorescence intensity (FI) of 50 cells from each sample was quantified using the Leica software and Image $\mathrm{J}$ software.

Mitochondrial Transmembrane Potential Assessment The changes in the mitochondrial membrane potential were measured with rhodamine 123 by confocal scanning microscopy. ${ }^{19)}$ The cells $\left(1 \times 10^{5} / \mathrm{mL}\right)$ were seeded on cover slips and cultured with vorinostat $(1.5 \mu \mathrm{M})$ and dasatinib $(4.5 \mu \mathrm{M})$ alone and in combination, for $12 \mathrm{~h}$. The cells were then washed with PBS and incubated with rhodamine $123(100 \mu \mathrm{L}, 10 \mu \mathrm{M})$ for $30 \mathrm{~min}$ at $37^{\circ} \mathrm{C}$. The fluorescent images were recorded by a confocal microscope (Leica TCS SP2) with 100×objective and excitation and emission settings of 505 and $520 \mathrm{~nm}$, respectively. The FI of 50 cells from each sample was quantified with the Leica software and Image $\mathrm{J}$ software.

Western Blot The cells $\left(1 \times 10^{5} / \mathrm{mL}\right)$ were seeded on $10-\mathrm{cm}$ dishes and cultured with vorinostat $(1.5 \mu \mathrm{M})$ and dasatinib $(4.5 \mu \mathrm{M})$, alone and in combination, for $12 \mathrm{~h}$. Then, the cells were harvested, lysed with the ice-cold RIPA lysis buffer containing $1 \mathrm{~mm}$ phenylmethylsulfonyl fluoride (PMSF) for $30 \mathrm{~min}$ at $4^{\circ} \mathrm{C}$ and centrifuged at $12000 \mathrm{rpm}$ for $10 \mathrm{~min}$ at $4^{\circ} \mathrm{C}$ to collect the liquid supernatant. The protein was quantified using a BCA Kit (Thermo), separated on sodium dodecyl sulfate-polyacrylamide gel electrophoresis (SDS-PAGE) gels at $14 \%$ polyacrylamide and blotted onto a polyvinylidene difluoride (PVDF) nitrocellulose membrane (Biosharp, China). Non-specific binding sites were blocked in 5\% skimmed milk powder in PBST for $1 \mathrm{~h}$ before probed with primary antibodies overnight at $4^{\circ} \mathrm{C}$. Antibodies were used at the following di- 
lutions: 1:1000 (Bcl-2 and $\beta$-actin), which were purchased from Cell Signaling Technology. They were incubated with horseradish peroxidase-conjugated immunoglobulin Gs (IgGs)
$(1: 5000)$ for $1 \mathrm{~h}$ at room temperature, and then visualized with enhanced chemiluminescence reagent (Millipore, U.S.A.).

Statistical Analysis All data are presented as means \pm
A

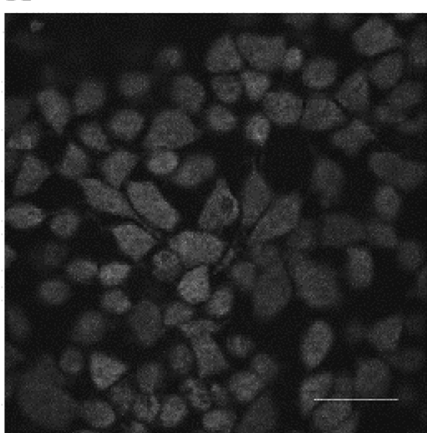

Control

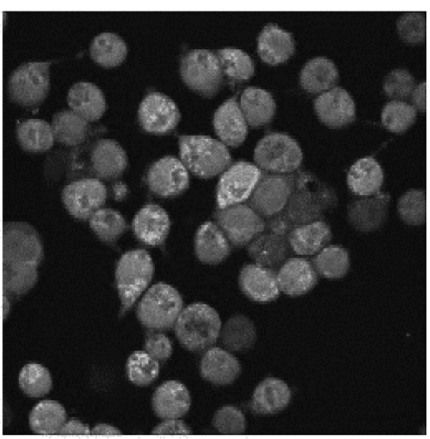

Dasatinib

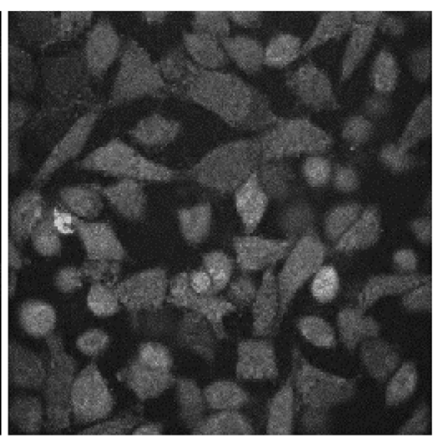

Vorinostat

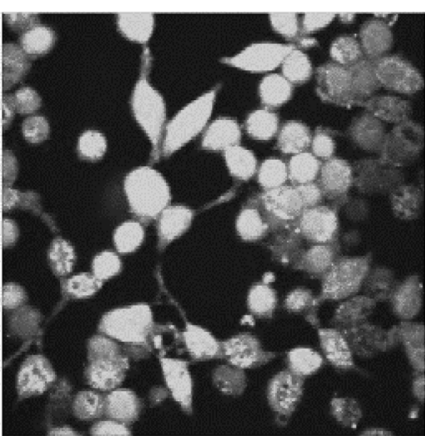

Vorinostat+dasatinib

C
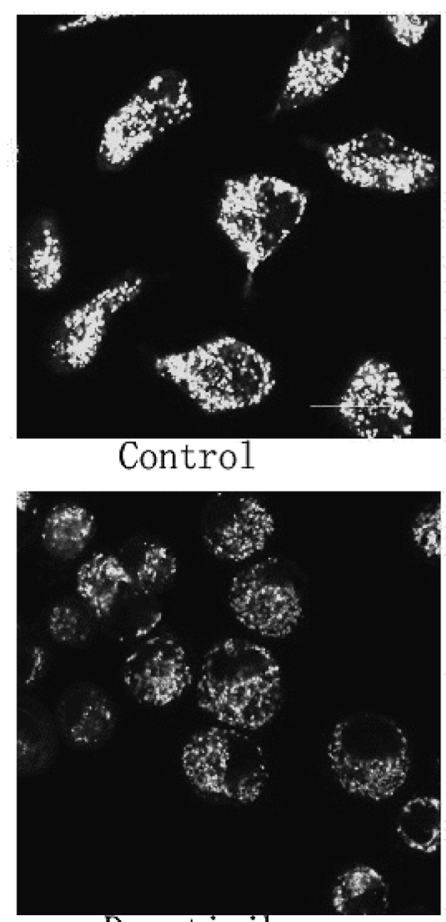

Dasatinib

B

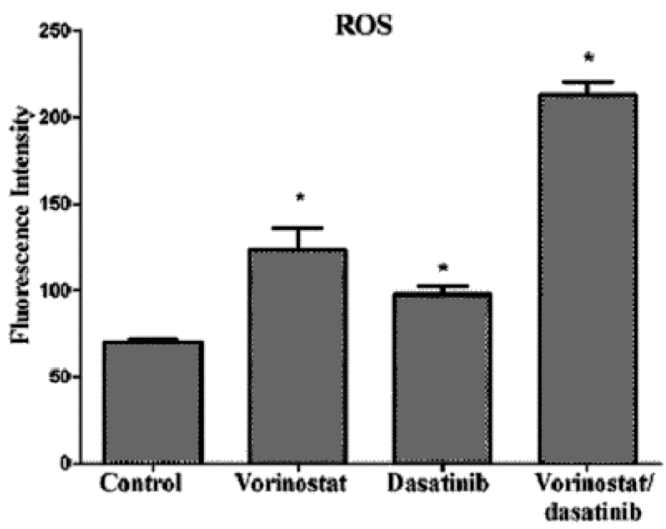

D

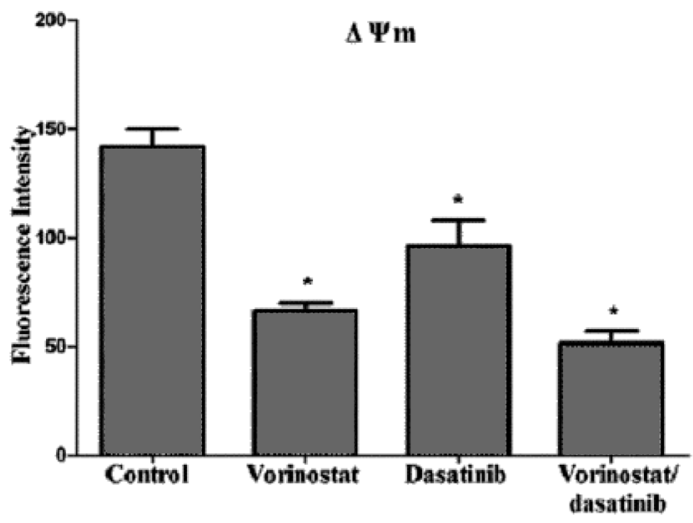

Fig. 3. Synergistic Induction of Intracellular Reactive Oxygen Species (ROS) Generation and Decrease Mitochondrial Membrane Potential ( $\Delta \Psi \mathrm{m})$ by the Combination of Vorinostat with Dasatinib in MCF-7 Cells

(A and B) ROS production was monitored in MCF-7 cells with DCF after treatment with vorinostat $(1.5 \mu \mathrm{M})$ and dasatinib (4.5 $\mu \mathrm{M})$, alone and in combination, for $12 \mathrm{~h}$. $(C$ and D) $\Delta \Psi \mathrm{m}$ changes were detected with Rhodamine 123 in MCF-7 cells after treatment with vorinostat $(1.5 \mu \mathrm{M})$ and dasatinib $(4.5 \mu \mathrm{M})$, alone and in combination, for $12 \mathrm{~h}$. FI was measured by laser scanning confocal microscopy. Data were presented as mean \pm S.D. from three independent experiments (B and D). $p<0.05$. $*$ Significantly different compared with the control group. 
A

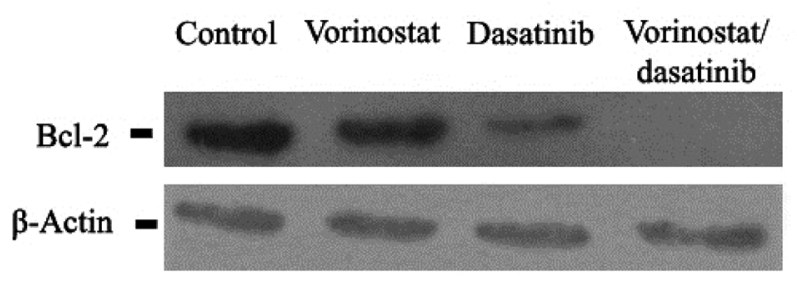

B

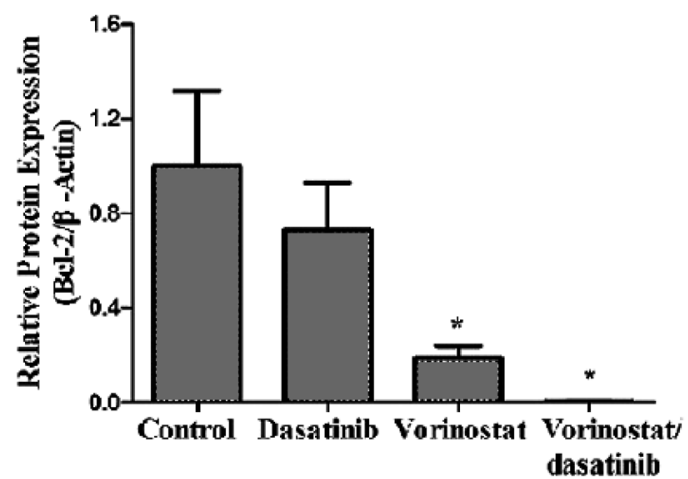

Fig. 4. Synergistic Decrease Bcl-2 Expression by the Combination of Vorinostat with Dasatinib in MCF-7 Cells

(A and B) The changes of Bcl-2 expression were detected by the western blots in MCF-7 cells after treatment with vorinostat $(1.5 \mu \mathrm{M})$ and dasatinib $(4.5 \mu \mathrm{M})$, alone and in combination, for $12 \mathrm{~h}$. Data were presented as mean \pm S.D. from three independent experiments (B). $p<0.05$. * Significantly different compared with the control group.

standard deviation (S.D.) from more than three independent experiments. SPSS16.0 and one-way ANOVA were used to analyze the data and $p<0.05$ was considered as statistically significant.

\section{RESULTS}

Synergistic Inhibition of MCF-7 Cell Proliferation by Vorinostat and Dasatinib We first evaluated the ability of vorinostat or dasatinib alone to inhibit growth of MCF-7 cells. The cells were treated with increasing concentrations of vorinostat or dasatinib and both the drugs showed dose-dependent cytotoxic effects in MCF-7 cells, with $\mathrm{Dm}\left(\mathrm{IC}_{50}\right)$ values of 6.32 and $21.06 \mu \mathrm{M}$, respectively. Considering the Chou-Talalay model requires drugs to be used at a fixed dose ratio, ${ }^{17)}$ we combined these drugs as the following ratios: vorinostat: dasatinib as 1:3 according to the Dm value ratios of $48 \mathrm{~h}$. The dose-effect relationship of vorinostat or dasatinib, alone and in combination, were evaluated on the growth of MCF-7 cells after exposure for $48 \mathrm{~h}$ (Figs. 1A-C). The median-effect analysis was conducted according to Chou's method. ${ }^{17)}$

CIs were calculated to determine the antitumor effects of the combinations; the plots of CI versus fraction affected (Fa) were shown in Figs. 1D and E. The combination of vorinostat and dasatinib exhibited a synergistic effect where the combination doses were 1.5 to $24 \mu \mathrm{M}$ at $48 \mathrm{~h}$ (Figs. 1C, D). As shown in Fig. 1E, the DRI values of vorinostat ranged from 2.34 to 4.18, whereas those of dasatinib ranged from 2.17 to 5.42 . Overall, the results of the MTT assay suggested that vorinostat and dasatinib could inhibit the proliferation of MCF-7 cells. The combinations of vorinostat with dasatinib $(1: 3)$, exhibited synergistic cytotoxic effects in MCF-7 cells.

Modulation of Cell Cycle Distribution and Induction of Apoptosis in MCF-7 Cells by Vorinostat and Dasatinib in Synergy The effects of vorinostat and dasatinib on cell cycle distribution were analyzed by the flow cytometry (Figs. 2A, B). Dasatinib induced a significantly accumulation of $\mathrm{G}_{0} / \mathrm{G}_{1}$ cells, together with a decrease in $\mathrm{S}$ and $\mathrm{G}_{2} / \mathrm{M}$ phases in MCF-7 cells $(p<0.05)$, compared with the control. In contrast, vorinostat caused a remarkable accumulation in $G_{2} / M$ phase cells at the expense of G1 phase cells. The combination of vorinostat with dasatinib, exhibited similar effects to that of vorinostat alone on cell cycle distribution $(p<0.05)$.

The apoptosis induced by vorinostat and dasatinib was determined through flow cytometry to further investigate the decreased viability of MCF-7 cells (Figs. 2C, D). Vorinostat and dasatinib alone can increase the proportion of apoptosis cells after treatment for $48 \mathrm{~h}(p<0.05)$. The combination of vorinostat with dasatinib caused a much higher apoptosis rate than those of drugs alone (36 vs. 16 and 12.57\%, $p<0.05$ ), indicating that the combination of dasatinib enhanced the apoptotic effect, especially early appotosis-induction of vorinostat significantly.

Synergistic Induction of Intracellular ROS Generation and Mitochondrial Transmembrane Potential $\left(\Delta \Psi_{m}\right)$ Loss by Vorinostat and Dasatinib The intracellular ROS generation was investigated using the fluorescent probe DCFH-DA to reveal that whether ROS was involved in mediating apoptosis induced by vorinostat and dasatinib. Treatment with vorinostat or dasatinib alone significantly enhanced the ROS generation in MCF-7 cells compared with the untreated cells $(p<0.05)$ (Figs. 3A, B). Interestingly, combined treatment with vorinostat and dasatinib remarkably promoted ROS generation (Figs. 3A, B).

The changes in $\Delta \Psi_{\mathrm{m}}$ have been linked to apoptosis ${ }^{20)}$; thus, we examined the influence of vorinostat and dasatinib on the $\Delta \Psi_{\mathrm{m}}$ of the MCF-7 cells with rhodamine 123. Rhodamine 123 is a mitochondria-binding specific cationic fluorescent dye that is proportional to the $\Delta \Psi_{\mathrm{m}}$. The results showed that incubation with vorinostat or dasatinib alone for $12 \mathrm{~h}$ led to a remarkable loss of $\Delta \Psi_{\mathrm{m}}$ in MCF-7 cells, revealed by the reduction of Rhodamine 123 FI compared with the control $(p<0.05)$ (Figs. $3 \mathrm{C}, \mathrm{D})$. The combination treatment of vorinostat and dasatinib showed a sharp decline of Rhodamine 123 FI to 51.34 compared with the control, vorinostat or dasatinib (96.36) alone after treatment for $12 \mathrm{~h}$.

Synergistic Decrease of Bcl-2 Expression by Vorinostat and Dasatinib $\mathrm{Bcl}-2$ is an inner mitochondrial membrane protein with an important role in preventing apoptosis. ${ }^{21)}$ Thus, we examined the effects of vorinostat and dasatinib, alone and in combination, on the Bcl-2 protein level. The results indicated that the combination treatment of vorinostat with dasatinib significantly decreased the expression of Bcl-2 after treatment for $12 \mathrm{~h}$ in the MCF-7 cells $(p<0.05)$ (Figs. 4A, B).

\section{DISCUSSION}

In this study, we found that the combination of vorinostat $(0.38$ to $6 \mu \mathrm{M})$ with dasatinib $(1: 3$ ratio) exerted a significant 
synergistic cytotoxic effects on MCF-7 cells in a dose-dependent manner. Furthermore, the combined treatment of HDAC inhibitor vorinostat and epidermal growth factor receptor (EGFR) inhibitor dasatinib synergistically induced apopotosis in MCF-7 cells.

As a HDAC inhibitor, vorinostat has been reported to induce cell cycle arrest, apopotosis, senescence, and differentiation in cancer cells. ${ }^{22}$ It has been reported that vorinostat combined with gefitinib have synergistic cytotoxicity in nonsmall cell lung cancers and hepatocarcinoma. ${ }^{723)}$ Our results showed that the $\mathrm{IC}_{50}$ of vorinostat is $6.32 \mu \mathrm{M}$, dasatinib is $21.06 \mu \mathrm{M}$, in human breast cancer cells MCF-7 after $48 \mathrm{~h}$. The results suggest that vorinostat sensitizes dasatinib-induced cytotoxicity in human breast cancer cells and reduces the doses of dasatinib by more than 2 times.

The combined beneficial effect can be achieved partly through cell cycle arrest, ROS production, and apoptosis induction in the mitochondria-mediated intrinsic pathway. Mitochondria have been considered as targets of anti-cancer drugs and have a key function in the regulation of apoptosis. ${ }^{24)}$

Our results indicated that vorinostat caused $\mathrm{G}_{2} / \mathrm{M}$ arrest, while dasatinib induced $\mathrm{G}_{1}$ arrest in MCF-7 cells. It is interesting that the combination of vorinostat and dasatinib led to an accumulation of cells in the $\mathrm{G}_{2} / \mathrm{M}$ phases at the expense of the $G_{1}$ phase. These findings suggested that vorinostat and dasatinib may be useful for controlling cancer cell growth because several lines of cancer cells have defects in the cell cycle checkpoints.

Mitochondria are the main production sites of ROS. The presence of excessive ROS in the mitochondria leads to the opening of the mitochondrial permeability transition pore, decline in $\Delta \Psi_{\mathrm{m}}$, and release of cytochrome $c$, which, in turn, trigger apoptosis. ${ }^{24}$ Several studies have provided evidence that vorinostat and dasatinib exhibit antitumor activity by inducing ROS generation and apoptosis. ${ }^{25-27)}$ Our results demonstrated that the incubation of MCF-7 cells with vorinostat and dasatinib, alone and in combination, increased the ROS generation, decreased $\Delta \Psi_{\mathrm{m}}$, down-regulated the anti-apoptosis protein Bcl-2 expression, and induced the mitochondria-mediated apoptosis.

In conclusion, the findings presented in this study demonstrated, for the first time, that vorinostat and dasatinib synergistically inhibited the growth of MCF-7 cells in vitro. This inhibition can be achieved partly by the generation of ROS and the decrease of Bcl-2 expression. Additional study is needed to elucidate the mechanistic interactions of combinatorial drugs. Moreover, the idea of combining epigenome-targeting drug with tyrosine kinase inhibitor should be given more attention, because it holds great potential in treating tumors with minimal side effects.

Acknowledgments This work was supported by the National Natural Science Foundation of China (No. 31100987) and the Natural Science Foundation of Shandong Province (No. ZR2016CM46).

Conflict of Interest The authors declare no conflict of interest.

\section{REFERENCES}

1) Ferlay J, Soerjomataram I, Dikshit R, Eser S, Mathers C, Rebelo M, Parkin DM, Forman D, Bray F. Cancer incidence and mortality worldwide: sources, methods and major patterns in GLOBOCAN 2012. Int. J. Cancer, 136, E359-E386 (2015).

2) Doan TB, Graham JD, Clarke CL. Emerging functional roles of nuclear receptors in breast cancer. J. Mol. Endocrinol., 58, R169R190 (2017).

3) Güth U, Myrick ME, Schötzau A, Kilic N, Schmid SM. Drug switch because of treatment-related adverse side effects in endocrine adjuvant breast cancer therapy: how often and how often does it work? Breast Cancer Res. Treat., 129, 799-807 (2011).

4) Locker GJ, Simonitsch I, Mader RM, Warlamides E, Gnant MF, Jakesz R, Rainer H, Steger GG. Cutaneous side effects in breast cancer patients treated with cytostatic polychemotherapy and rh GM-CSF: immune phenomena or drug toxicity? Breast Cancer Res. Treat., 34, 213-219 (1995).

5) Mao JJ, Chung A, Benton A, Hill S, Ungar L, Leonard CE, Hennessy S, Holmes JH. Online discussion of drug side effects and discontinuation among breast cancer survivors. Drug Saf., 22, 256-262 (2013).

6) Chen D, Liu X, Yang Y, Yang H, Lu P. Systematic synergy modeling: understanding drug synergy from a systems biology perspective. BMC Syst. Biol., 9, 56 (2015).

7) Jeannot V, Busser B, Vanwonterghem L, Michallet S, Ferroudj S, Cokol M, Coll JL, Ozturk M, Hurbin A. Synergistic activity of vorinostat combined with gefitinib but not with sorafenib in mutant KRAS human non-small cell lung cancers and hepatocarcinoma. Onco Targets Ther., 9, 6843-6855 (2016).

8) Farhat FS, Temraz S, Kattan J, Ibrahim K, Bitar N, Haddad N, Jalloul R, Hatoum HA, Nsouli G, Shamseddine AI. A phase II study of lipoplatin (liposomal cisplatin)/vinorelbine combination in HER-2/neu-negative metastatic breast cancer. Clin. Breast Cancer, 11, 384-389 (2011).

9) Liu XH, Man YN, Cao R, Liu C, Wu XZ. Individualized chemotherapy based on organ selectivity: a retrospective study of vinorelbine and capecitabine for patients with metastatic breast cancer. Curr. Med. Res. Opin., 30, 1017-1024 (2014).

10) Baylin SB, Jones PA. A decade of exploring the cancer epigenome - biological and translational implications. Nat. Rev. Cancer, 11, 726-734 (2011)

11) Rodriguez R, Miller KM. Unravelling the genomic targets of small molecules using high-throughput sequencing. Nat. Rev. Genet., 15, 783-796 (2014).

12) Glozak MA, Seto E. Histone deacetylases and cancer. Oncogene, 26, 5420-5432 (2007).

13) Gong F, Miller KM. Mammalian DNA repair: HATs and HDACs make their mark through histone acetylation. Mutat. Res., 750, 23-30 (2013).

14) Okabe S, Tauchi T, Kimura S, Maekawa T, Kitahara T, Tanaka Y, Ohyashiki K. Combining the ABL1 kinase inhibitor ponatinib and the histone deacetylase inhibitor vorinostat: a potential treatment for BCR-ABL-positive leukemia. PLOS ONE, 9, e89080 (2014).

15) Mosmann T. Rapid colorimetric assay for cellular growth and survival: application to proliferation and cytotoxicity assays. J. Immunol. Methods, 65, 55-63 (1983).

16) Chou TC, Talalay P. Quantitative analysis of dose-effect relationships: the combined effects of multiple drugs or enzyme inhibitors. Adv. Enzyme Regul., 22, 27-55 (1984).

17) Chou TC. Theoretical basis, experimental design, and computerized simulation of synergism and antagonism in drug combination studies. Pharmacol. Rev., 58, 621-681 (2006).

18) Engelmann J, Volk J, Leyhausen G, Geurtsen W. ROS formation and glutathione levels in human oral fibroblasts exposed to TEGDMA and camphorquinone. J. Biomed. Mater. Res. B Appl. 
Biomater., 75B, 272-276 (2005).

19) Scaduto RC Jr, Grotyohann LW. Measurement of mitochondrial membrane potential using fluorescent rhodamine derivatives. Biophys. J., 76, 469-477 (1999).

20) Ly JD, Grubb DR, Lawen $A$. The mitochondrial membrane potential $\left(\Delta \Psi_{\mathrm{m}}\right)$ in apoptosis; an update. Apoptosis, 8, 115-128 (2003).

21) Wei H, Kang B, Wei W, Liang G, Meng QC, Li Y, Eckenhoff RG. Isoflurane and sevoflurane affect cell survival and BCL-2/BAX ratio differently. Brain Res., 1037, 139-147 (2005).

22) Grant S, Dai Y. Histone deacetylase inhibitors and rational combination therapies. Adv. Cancer Res., 116, 199-237 (2012).

23) Han JY, Lee SH, Lee GK, Yun T, Lee YJ, Hwang KH, Kim JY, Kim HT. Phase I/II study of gefitinib (Iressa ${ }^{B}$ ) and vorinostat (IVORI) in previously treated patients with advanced non-small cell lung cancer. Cancer Chemother. Pharmacol., 75, 475-483 (2015).

24) Hockenbery DM. Targeting mitochondria for cancer therapy. Envi- ron. Mol. Mutagen., 51, 476-489 (2010).

25) Leone A, Roca MS, Ciardiello C, Terranova-Barberio M, Vitagliano C, Ciliberto G, Mancini R, Di Gennaro E, Bruzzese F, Budillon A. Vorinostat synergizes with EGFR inhibitors in NSCLC cells by increasing ROS via up-regulation of the major mitochondrial porin VDAC1 and modulation of the c-Myc-NRF2-KEAP1 pathway. Free Radic. Biol. Med., 89, 287-299 (2015).

26) Okon IS, Coughlan KA, Zhang M, Wang Q, Zou MH. Gefitinibmediated reactive oxygen specie (ROS) instigates mitochondrial dysfunction and drug resistance in lung cancer cells. J. Biol. Chem., 290, 9101-9110 (2015).

27) Xue T, Luo P, Zhu H, Zhao Y, Wu H, Gai R, Wu Y, Yang B, Yang $\mathrm{X}, \mathrm{He} \mathrm{Q}$. Oxidative stress is involved in Dasatinib-induced apoptosis in rat primary hepatocytes. Toxicol. Appl. Pharmacol., 261, 280-291 (2012). 